\title{
EVALUACIÓN DE LA PERTINENCIA DE UN PROGRAMA DE CULTURA FÍSICA, DEPORTE Y RECREACIÓN DE UNA UNIVERSIDAD PRIVADA EN COLOMBIA
}

\author{
${ }^{1}$ Mike William Barreto Becerra, ${ }^{2}$ Paula Camila Ramírez Muñoz \\ ${ }^{1}$ Profesional en Cultura Física, Deporte y Recreación, Ph.D. (c) en Ciencias de la Actividad Física y el Deporte. Universidad Santo Tomás, Bucaramanga \\ ${ }^{2}$ Fisioterapeuta, magíster en Epidemiología, Universidad Santo Tomás, Bucaramanga
}

Autor responsable de correspondencia: Mike William Barreto Becerra

Correoelectrónico:mike.barreto@ustabuca.edu.co

\section{RESUMEN}

La pertinencia de una universidad o de un programa educativo es la forma como esta responde a las necesidades de la sociedad y de su contexto. Una de las maneras de hacerlo es mediante el desempeño de sus graduados, por ello, la permanente comunicación universidad-graduado le permite a la institución retroalimentarse y asumir los cambios que surgen en el contexto con mayor precisión y dinamismo.

Objetivos: evaluar la pertinencia del programa académico de la Facultad de Cultura Física, Deporte y Recreación de una universidad privada de Bucaramanga desde la perspectiva de sus graduados y su empleabilidad en el mercado laboral.

Metodología: se realizó un estudio observacional, analítico, de corte transversal, orientado a conocer la percepción acerca de la formación y desempeño laboral de los graduados del Programa de la Facultad de Cultura Física, Deporte y Recreación de la Universidad Santo Tomás de Bucaramanga. Se hicieron encuestas utilizando el instrumento del Observatorio Laboral de Educación (OLE) y se implementó el modelo propuesto por Beltrán y colaboradores en el 2011, para asumir la ruta de seguimiento a graduados. La muestra estuvo constituida por 63 graduados desde el 2000 hasta el 2012. Se exploró asociación entre variables mediante las pruebas Chi ${ }^{2}$ para las variables cualitativas, y prueba de Kruskal-Wallis para variables cuantitativas no paramétricas.

Resultados: se pudo determinar que los participantes del estudio cumplieron con sus expectativas iniciales al culminar sus estudios y lograron realizar una inserción laboral temprana y conforme con sus competencias y campo de formación.

Conclusión: Se evidencia una clara misión de la Universidad por sus propósitos de imprimir en sus egresados los valores y la ética profesional, considerando la formación como compromiso con la sociedad, declarando también su perfil desde los referentes disciplinares y profesionales. [Barreto MW, Ramírez PC. Evaluación de la pertinencia de un programa de Cultura Física, Deporte y Recreación de una universidad privada en Colombia. Ustasalud. 2015;14:40-47].

Palabas clave: Evaluación, enseñanza superior, administración.

\section{EVALUATION OF THE RELEVANCE OF A PROGRAM OF PHYSICAL CULTURE, SPORTS AND RE- CREATION OF A PRIVATE UNIVERSITY IN COLOMBIA}

\section{ABSTRACT}

The relevance of a university or an educational program is how it responds to the needs of society and its context. One of the ways to do this is through the performance of its graduates, a permanent university-graduate communication allows the institution to receive feedback and to assume the changes that arise in the context with greater precision and dynamism.

Objectives: evaluate the relevance of the academic program of the Faculty of Physical Culture, Sports and Recreation of a private university of Bucaramanga from the perspective of its graduates and its employability in the working market.

Methodology: an observational, analytical and cross-sectional study was conducted, aimed at knowing the perception about the training and work performance of the graduates of the program of the Faculty of Physical Culture, Sports and Recreation of the University of Santo Tomás de Bucaramanga. Surveys were carried out using the instrument of the Observatorio Laboral de Educación de Colombia (OLE) and the model proposed by Beltrán and collaborators was implemented, to assume the follow-up path to graduates. The sample consisted of 63 graduates from the year 2000 to 2012. The association between variables was explored through the Chi2 tests for the qualitative variables and the Kruskal-Wallis test for nonparametric quantitative variables.

Results: it was observed that the study participants fulfilled their initial expectations upon completing their studies and achieved an early working insertion in accordance with their competencies and training field.

Conclusion: A clear mission of the University is evident for its purposes of spreading ethical professional values on the graduates, also since considers the education as a community commitment and states its profile from disciplinary and professional references.

Keywords: Educational assessment, graduate education, administration.

Recibido para publicación: 18 de junio de 2015. Aprobado para publicación: 23 de septiembre de 2015. 


\section{INTRODUCCIÓN}

Los nuevos escenarios políticos, sociales y económicos nacionales e internacionales, exigen cada vez mayores estándares de calidad y de pertinencia de la educación universitaria, haciendo que estas organizaciones deban adaptarse para responder a las necesidades de la sociedad, en coherencia con sus diferentes niveles educativos, incluyendo la educación para el trabajo y el desarrollo humano, y así responder a la dinámica social y mantener el liderazgo en los procesos de desarrollo de los países a través de sus propuestas académicas ${ }^{1}$.

La pertinencia es percibida como la contribución que la universidad hace a su contexto social, cultural, económico, político, fundamentado en el desarrollo científico, aspectos necesarios para el estudio, seguimiento y evaluación, según el Ministerio de Educación Nacional ${ }^{2}$. También el Plan Decenal de Educación señala la importancia de diseñar currículos pertinentes, orientados hacia el desarrollo de las dimensiones del ser, saber, hacer y convivir en la construcción e identidad nacional y la cultura, mediante los procesos de aprendizaje, investigación y la permanencia en el sistema.

La evaluación de los programas de pregrado hace parte de una estrategia del Estado colombiano por mejorar los estándares de calidad de la educación superior; para este fin, los procesos de autoevaluación que se planifican en el interior de cada una de las universidades les permite ganar un posicionamiento en la sociedad, reconociéndolas por sus buenas prácticas, garantizando la prestación de un servicio educativo acorde con las necesidades actuales de la sociedad. Desde esta perspectiva, los graduados, juegan un papel fundamental, pues son quienes permiten conocer a través del impacto de su desempeño, la pertinencia del programa ${ }^{3}$.

Esta investigación tiene por objeto conocer la percepción de los graduados respecto a la pertinencia del programa de la Facultad de Cultura Física, Deporte y Recreación, siendo ellos el producto entregado al contexto como resultado final de un proceso, para lo cual se hace necesario hacer uso del Modelo de Evaluación de Programas CIPP (contexto, entrada, proceso y producto), postulado por Daniel Stufflebeam ${ }^{4}$, que permite establecer información acerca de la eficiencia y eficacia del programa, de tal manera que pueda conocer la pertinencia del mismo y a partir de sus resultados conocer, analizar y tomar decisiones para el planteamiento de acciones de mejora hacia los estándares de calidad ${ }^{5}$.

\section{MATERIALES Y MÉTODOS}

Se realizó un estudio observacional, analítico de corte transversal ${ }^{6}$. La población incluyó a todos los graduados desde el 2000 hasta el 2012. La totalidad de graduados del programa era de 220 , quienes fueron contactados vía telefónica, por Internet y mediante visitas en sus hogares y sitios de trabajo. La tasa de respuesta fue del $28,6 \%$, es decir, se logró encuestar a 63 participantes.

Se aplicó una encuesta cara a cara para garantizar su adecuado diligenciamiento, disminuyendo las posibilidades de error en la información registrada. Se realizaron encuestas utilizando el instrumento del Observatorio Laboral de Educación (OLE), estructuradas en ocho partes correspondientes a información personal y familia (relacionados con el desarrollo personal y principales características); historia académica y financiera (datos socioeconómicos); información sobre las competencias para conocer su desempeño; información sobre el plan de vida; situación laboral y el mercado laboral del graduado; nivel de identidad con la institución de educación superior; satisfacción de los recursos ofrecidos por la institución de donde se graduó y, finalmente, los datos fundamentales para el seguimiento a graduados ${ }^{7}$.

Se aplicaron 66 encuestas para los cuatro momentos previstos en el Observatorio Laboral de Educación del $\mathrm{MEN}^{7}$, a los graduados según el momento 0 (recién graduados), momento 1 (graduados hace 1 año), momento 3 (graduados hace 3 años) y momento 5 (graduados hace 5 años).

Para la información se realizó un análisis univariado, teniendo en cuenta la naturaleza y distribución de las variables, aplicando medidas de tendencia central (media y mediana) y de dispersión (desviación típica o estándar), para variables cuantitativas y para variables cualitativas se presentan tablas de frecuencia. Se evaluó la normalidad de las variables continuas mediante la prueba de Kolmogorov Smirnov; adicionalmente, se realizó análisis bivariado para identificar la correlación entre variables mediante correlación de Pearson y se exploró asociación entre variables mediante las pruebas $\mathrm{Chi}^{2}$ para las variables nominales, prueba de Kruskal-Wallis para variables ordinales (o no paramétricas) por tratarse de cuatro grupos de comparación correspondientes a cada momento de los egresados. Para la comparación por sexos se utilizó la prueba U de Mann-Whitney como prueba no paramétrica. La sistematización de la información y el análisis se realizaron mediante el software SPSS 21 de IBM ${ }^{\circledR}$.

Los autores declaran que no hubo ningún conflicto de interés en el desarrollo de este proyecto. Los 
procedimientos aplicados en esta investigación se ajustan a las consideraciones de la Resolución 008430 del 93. Es un estudio sin riesgo, los participantes firmaron consentimiento informado $\mathrm{y}$ los datos obtenidos resultado de las encuestas de seguimiento a graduados del OLE se mantuvieron en confidencialidad.

\section{RESULTADOS}

Se realizó una evaluación de la pertinencia del programa de la Facultad de Cultura Física, Deporte y Recreación desde la perspectiva de los graduados, en cuatro momentos de tiempo que corresponden al momento del grado, uno, tres y cinco años después de egresados.

En total se encuestaron 63 egresados en los cuatro momentos (Tabla 1). De estos, el 92,1\% $(n=58)$ correspondieron al género masculino y en el $61,9 \%$ $(\mathrm{n}=39)$ el estado civil era soltero. El promedio de edad fue 28,7 (DS 3,9 años), rango comprendido entre 22 a 39 años.

Al realizar la comparación de la situación laboral actual de los egresados en los momentos en que se aplicó la encuesta, se observó un comportamiento diferencial entre el momento cero y los demás momentos; esto, teniendo en cuenta que los graduados de recién egreso aún no se encontraban desvinculados de la Universidad. Por tanto, como se observa en la Figura 1, la mayoría de los encuestados en el momento cero contestó que se encontraban estudiando (66,7\%). Por el contrario, en los tres momentos posteriores ( 1 a 3 ) se observa que más de la tercera parte se encontraba vinculado laboralmente (75\%, $88,9 \%$ y $76,2 \%$, respectivamente).

De igual forma, al comparar las respuestas de los egresados en los cuatro momentos, respecto a la realización de alguna actividad remunerada adicional a la actividad principal, se observó que solamente en el momento dos se presentó una frecuencia alta el poseer una empresa o negocio $(62,5 \%)$ y en los otros momentos fueron frecuencias menores al $20 \%$. De otra parte, para los encuestados del momento uno, el $60 \%$ se encontraba trabajando como empleado; al igual que el $45,5 \%$ de los encuestados del momento tres.

Respecto al desempeño de la actividad que realizan, se observó que los egresados encuestados en los cuatro momentos, son en su mayoría empleados de empresa particular o del gobierno (>64,7\%), los trabajadores independientes en general correspondieron a menos del 30\% y los empresarios o empleadores solamente se encontraron en el momento dos (5,9\%) (Figura 2).
Tabla 1. Participantes en el estudio de acuerdo con los momentos de aplicación de la encuesta OLE

\begin{tabular}{lccc}
\hline \multicolumn{1}{c}{$\begin{array}{c}\text { Momento de } \\
\text { aplicación de la } \\
\text { encuesta OLE }\end{array}$} & Año grado & $\begin{array}{c}\text { Participantes } \\
\text { (n) }\end{array}$ & $\%$ \\
\hline $\begin{array}{l}\text { Momento del } \\
\text { grado (Momento 0) }\end{array}$ & 2012 & 15 & 23,8 \\
$\begin{array}{l}\text { Un año después } \\
\text { del grado } \\
\text { (Momento 1) }\end{array}$ & 2011 & 11 & 17,5 \\
$\begin{array}{l}\text { Tres años después } \\
\text { del grado } \\
\text { (Momento 2) }\end{array}$ & 2009 & 17 & 26,9 \\
$\begin{array}{l}\text { Cinco años } \\
\text { después del grado } \\
\text { (Momento 3) }\end{array}$ & 2007 & 20 & 31,7 \\
TOTAL & & & \\
\hline
\end{tabular}

En ese sentido, también se observó, que el empleo de más del $85 \%$ de los encuestados se encuentra directamente relacionado con la carrera que estudiaron y los demás empleos están indirectamente relacionados con esta.

Respecto al promedio de ingresos en el mes anterior, se observó que a medida que los egresados cuentan con mayor experiencia laboral, el promedio de los ingresos va aumentando. De igual forma, al realizar el análisis estadístico de los promedios de ingresos reportados, se observó en la prueba de Kruskal-Wallis que existe una diferencia estadísticamente significativa entre los ingresos de los cuatro momentos $(p=0,001)$, Figura 4 .

Se observó que el promedio de ingresos mensuales fue más elevado para hombres que para mujeres, siendo de $\$ 1.658 .649$ pesos colombianos y $\$ 846.000$, respectivamente, y la diferencia fue estadísticamente significativa con $\mathrm{p}=0,0025$ ( $\mathrm{U}$ de Mann-Whitney).

El ámbito de las actividades de la empresa donde laboran los egresados fue predominantemente local (25\% a $75 \%$ ) en los cuatro momentos, seguido del nacional $(16,7 \%$ a $50 \%)$; y resaltándose que en el ámbito internacional, solamente fue reportado en el momento dos donde se encontró un $15,4 \%$ (Figura 5). Sin embargo, respecto al vínculo entre la organización donde laboran y la Universidad, los resultados tuvieron un comportamiento diferencial entre el momento uno y los demás momentos. En el momento uno todos manifestaron la existencia de dicho vínculo, por el contrario, en los otros momentos se reportó solo entre el $25 \%$ y el $42,9 \%$. 


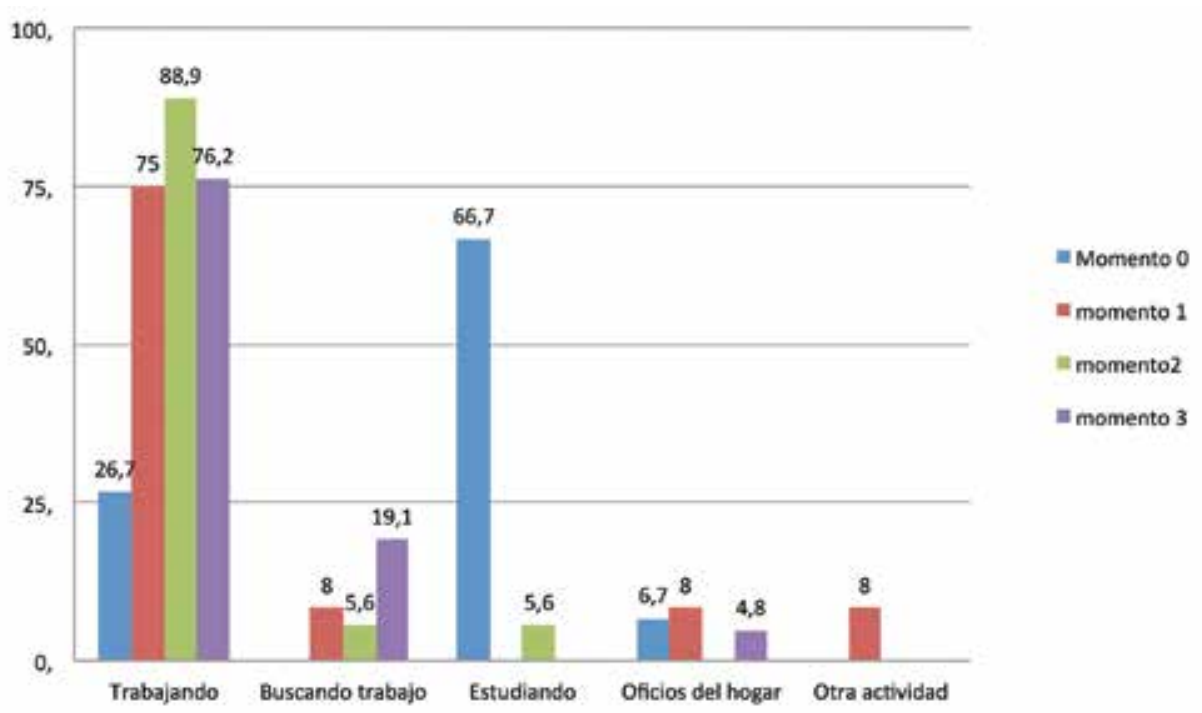

Figura 1. Actividad que ocupa la mayor parte del tiempo de los egresados encuestados en los cuatro momentos.

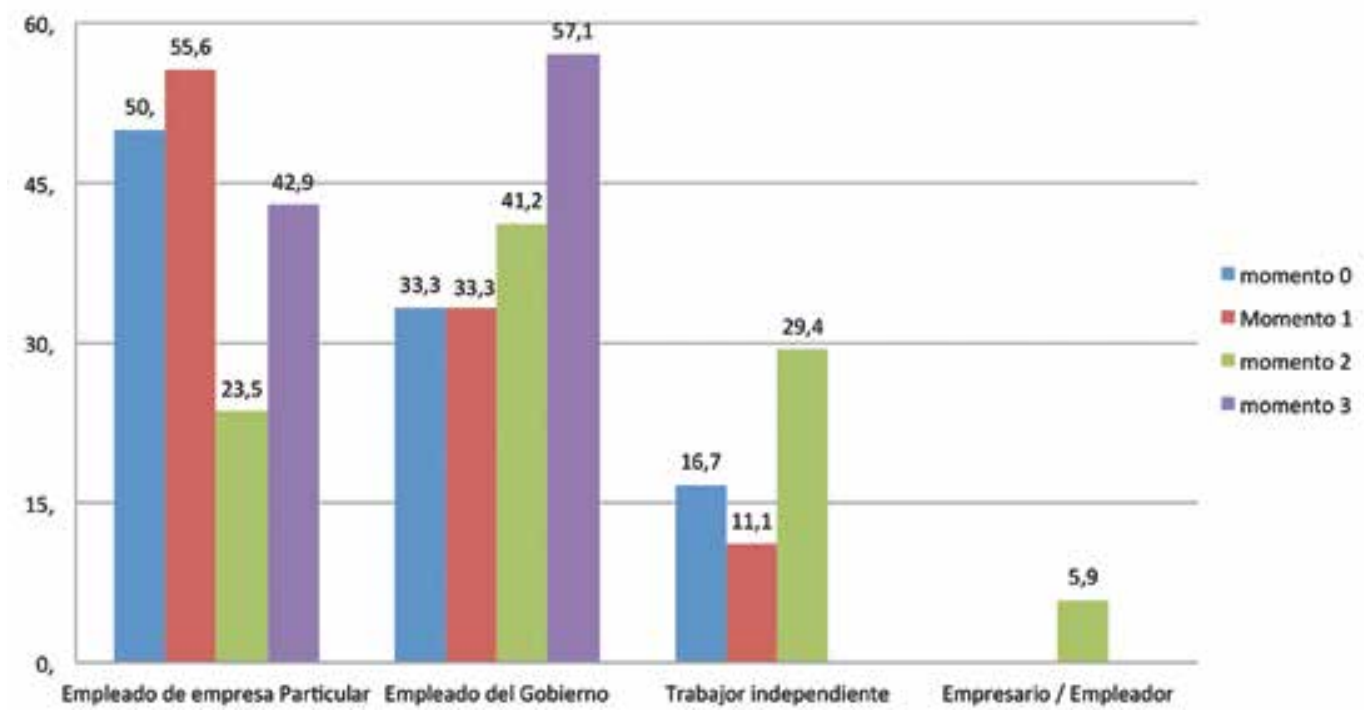

Figura 2. Tipo de vinculación laboral de los egresados, en los cuatro momentos de evaluación.

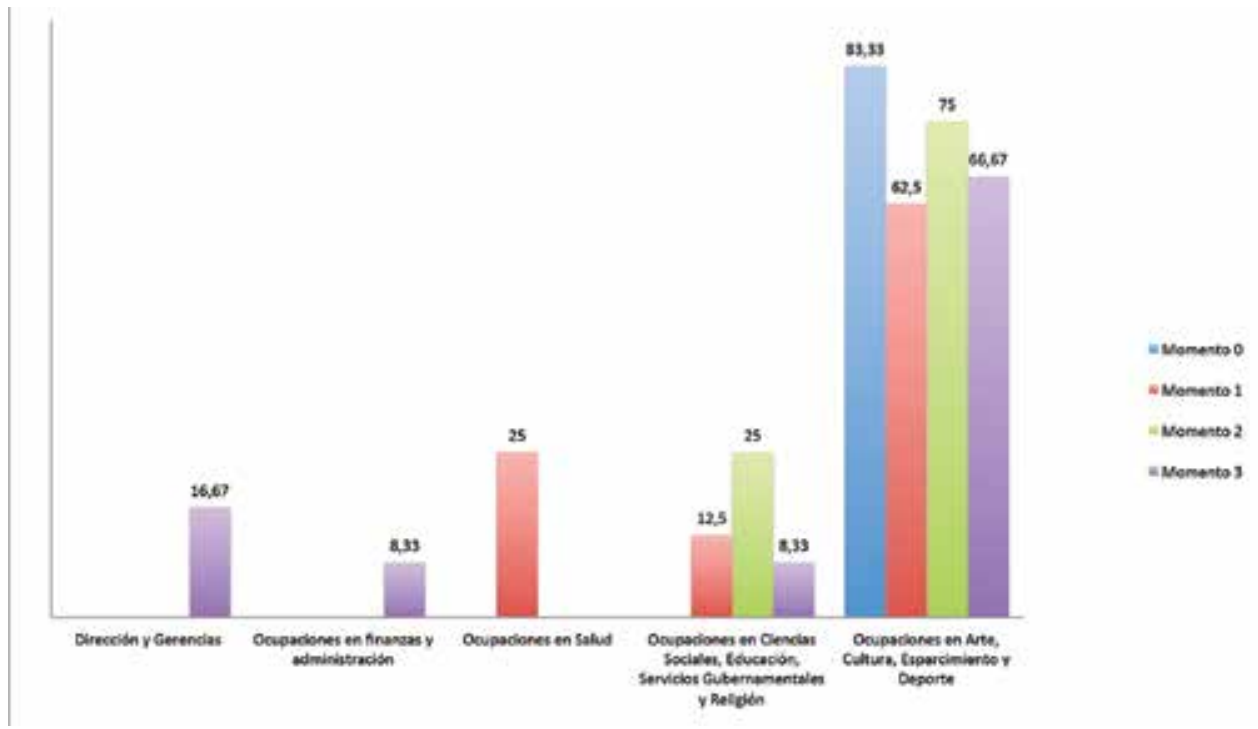

Figura 3. Ocupación actual de los egresados en los cuatro momentos de evaluación. 


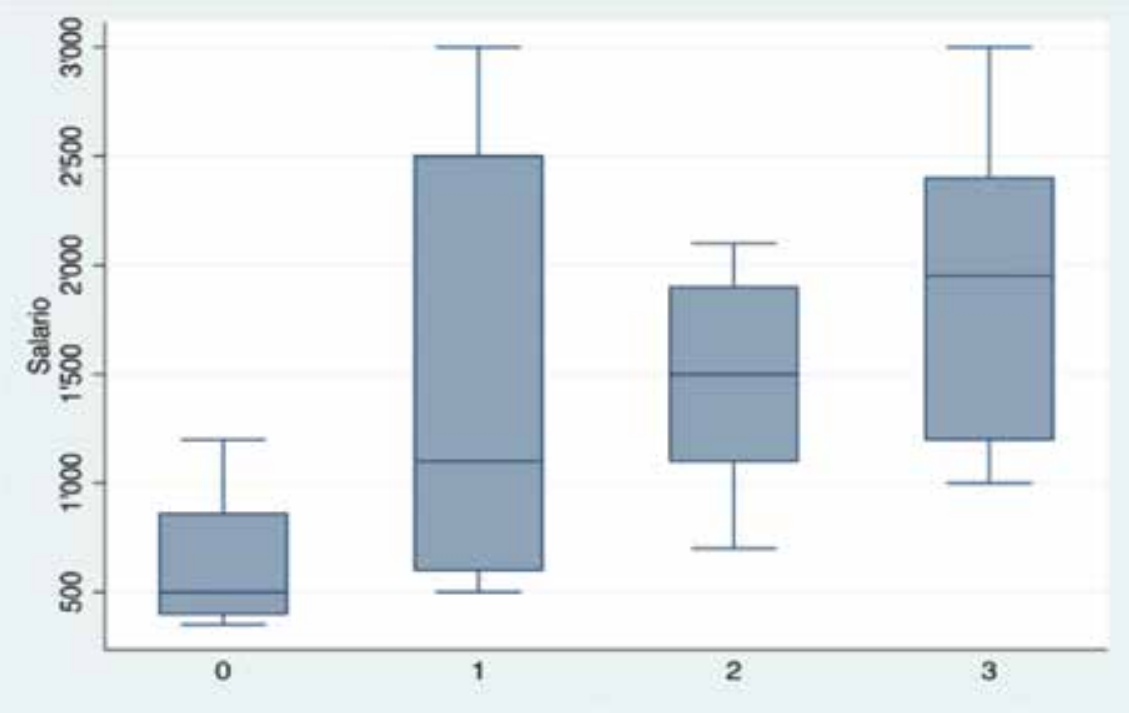

Figura 4. Promedio de ingresos en el mes anterior de los graduados, en los cuatro momentos de evaluación.

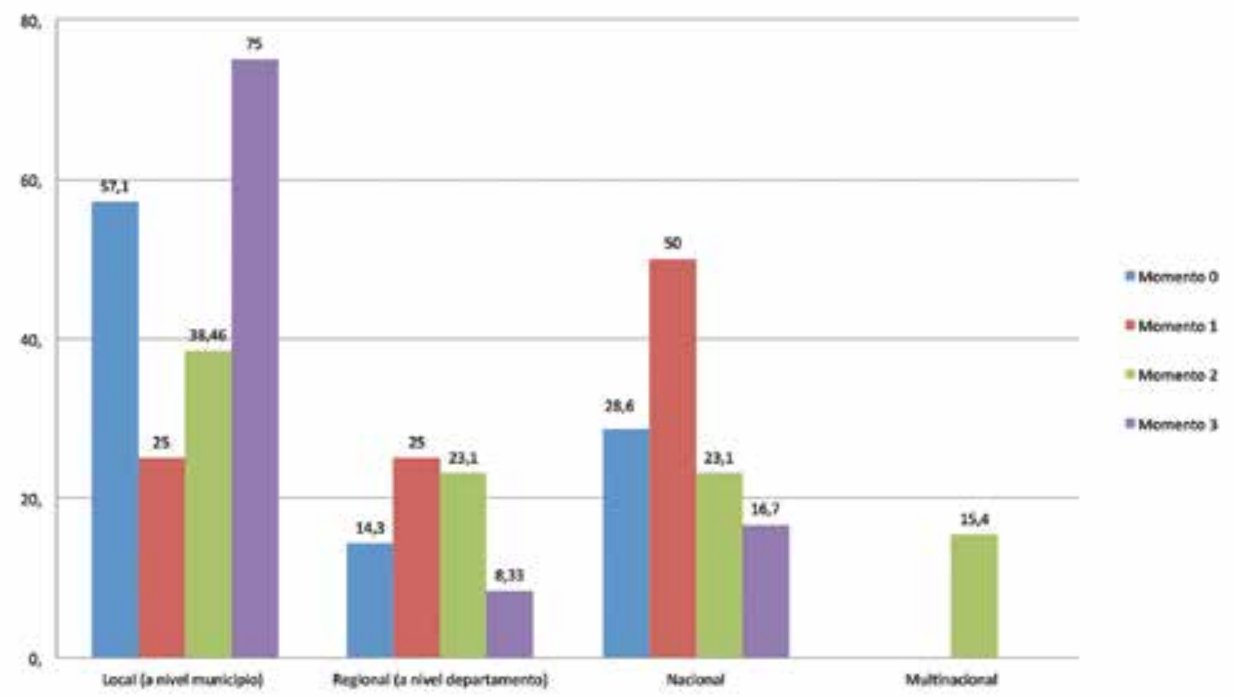

Figura 5. Ámbito de las actividades de la empresa donde laboran los graduados, de acuerdo con el momento de evaluación.

Se debe resaltar que en cuanto a la competencia escogida como la más fuerte entre los egresados, se obtuvieron resultados diferentes en cada uno de los momentos; de esta forma, en el momento uno fue "utilizar el tiempo de manera efectiva" $(41,7 \%)$; en el momento dos se encuentran "ser creativo e innovador" $(11,1 \%)$ y "aplicar valores y ética profesional en el desempeño laboral" $(11,1 \%)$, entre otros; de igual forma, en el momento tres se escogió esta última con un 35\% de evaluaciones favorables.

Por el contrario, para la competencia más débil, en los tres momentos los egresados coincidieron en escoger "utilizar herramientas informáticas especializadas" con $41,7 \%$ en el momento uno, $38,9 \%$ en el momento dos y $30 \%$ en el momento tres.

Igualmente, se evaluó en dos momentos, la competencia adquirida durante la formación, que de acuerdo con la percepción de los egresados le ha sido de mayor utilidad en su ejercicio profesional; de esta forma, se observó que para los encuestados en el momento dos fue "planificar y utilizar el tiempo de manera efectiva de tal forma que se logran los objetivos planteados" $(16,7 \%)$ y "aplicar valores y ética profesional en el desempeño laboral" (16,7\%); de otra parte para los egresados encuestados en el momento tres fue "asumir una cultura de convivencia" (20\%).

También se encontró que la competencia que les ha sido de menor utilidad en su desempeño laboral fue "trabajar bajo presión" con $26,7 \%$ y $23,5 \%$ para los momentos dos y tres, respectivamente.

Finalmente, respecto a las posibilidades laborales derivadas de la condición de egresado de la Universidad Santo Tomás de Bucaramanga, el 66,7\% consideró que estas eran moderadas a buenas (Figura 6). 


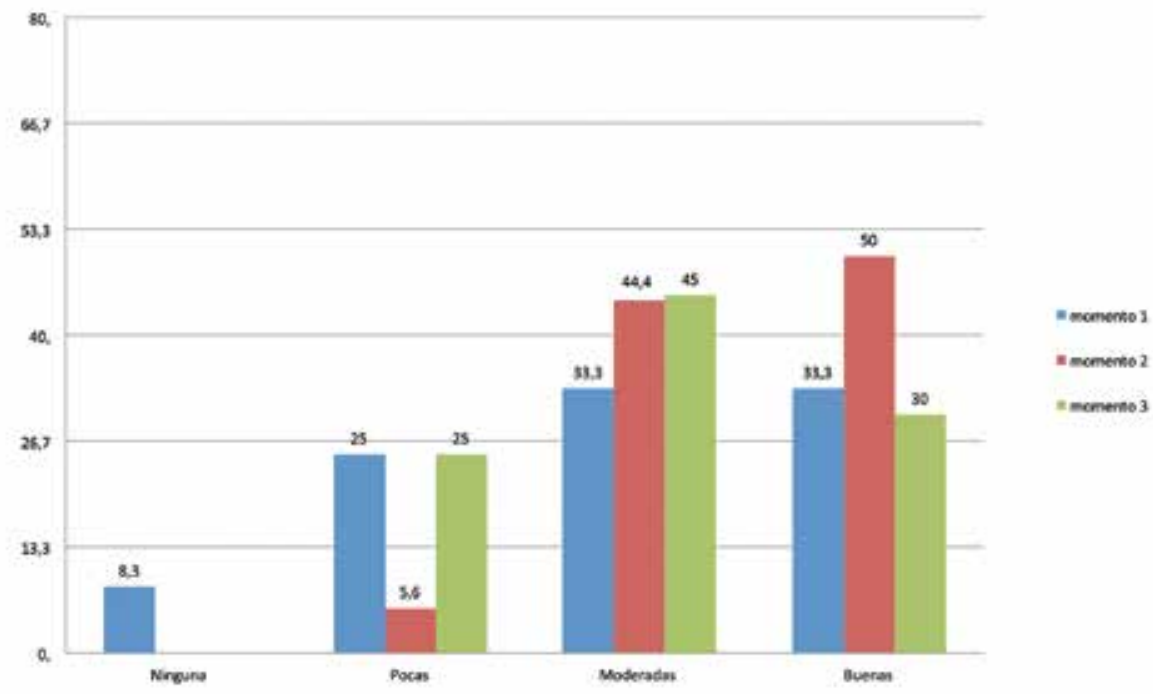

Figura 6. Posibilidades laborales derivadas de la condición de graduado de la USTA.

Debido a la relación encontrada entre el ingreso y el momento en que se encontraban los egresados, se exploró si existía correlación entre la edad y las horas semanales de trabajo (ambas variables con distribución normal, valor de $\mathrm{p}>0,05$ ); sin embargo, el coeficiente de correlación de Pearson fue de $(\mathrm{r}=0,22)$ de acuerdo con la interpretación sugerida, la correlación es baja.

Se exploró si existía asociación entre poseer trabajo y cada uno de los momentos, mediante la prueba de $\mathrm{Chi}^{2}$, sin embargo no hubo evidencia de dicha asociación $(p=0,553)$. Adicionalmente se realizó dicha comparación por sexos, encontrando que en el momento de la encuesta existió una diferencia estadísticamente significativa entre estar trabajando actualmente y el sexo, pues habían más mujeres en condición de desempleo que hombres $(\mathrm{p}=0,022)$.

\section{DISCUSIÓN}

Por medio de la presente investigación se buscó conocer la pertinencia del programa académico de la Facultad de Cultura Física, Deporte y Recreación de una universidad privada, desde la perspectiva de sus graduados.

Los graduados de los diferentes momentos cuentan con trabajo, resultado de las competencias obtenidas en la universidad; sin embargo, en el momento 0 que son los estudiantes activos de último semestre no reportaron ocupación laboral, esto debido a que en su último semestre se encuentran finalizando con trabajos, prácticas y proyectos de investigación que ocupan su tiempo, como también fue comprobado por los autores Fleming, Martin y Hughes ${ }^{8}$ en su trabajo acerca de la importancia de las prác- ticas de los estudiantes que les permitan obtener escenarios donde se fortalezcan sus habilidades, en particular para el manejo del tiempo, el trabajo en equipo, la iniciativa y la autosuficiencia.

Es importante mencionar que la proliferación de nuevas actividades deportivas y recreativas han permitido una mayor demanda de profesionales del área del deporte y la recreación en la ciudad y, en general, en todo el departamento de Santander, donde una de las fuentes y estrategias de competitividad creciente está relacionada con el turismo, dada la demanda de guías turísticos y profesionales que se hagan cargo de las actividades deportivas y recreativas de los deportes de aventura y extremos. Comparable con el Libro Blanco Título de grado de ciencias de la actividad fisica y el deporte, de la Agencia Nacional de Evaluación de la Calidad y Acreditación ANE$\mathrm{CA}^{9}$, donde menciona la importancia del quehacer profesional en el campo de la recreación, el ocio y el turismo, en particular en el medio natural y rural y señala estas áreas como temáticas potenciales de formación a nivel posgradual.

En el tema de la inserción laboral para los cuatro momentos se pudo comprobar que los egresados se encuentran trabajando en empresas que se relacionan con la carrera estudiada, esto permite una mayor satisfacción desde la percepción de los estudiantes y se puede explicar por el modelo educativo de la Universidad, el programa de estudios y las prácticas pedagógicas.

Es evidente que la oferta laboral para los egresados en la región y sus municipios es alta, que bien puede ser un indicador de la necesidad a la cual responde la universidad y la comprobación de la pertinencia del programa, puesto que los graduados cuentan 
con un buen nivel de empleabilidad ${ }^{10,11}$. Quiere decir que los graduados una vez terminada su carrera logran hacer una inserción laboral rápida en el mercado regional en el campo disciplinar ${ }^{1,2,10,11}$.

La mayoría son empleados de empresas particulares y cuentan con una vinculación laboral variada, acorde a las normativas legales colombianas. También es importante resaltar que el medio de búsqueda de trabajo más usado fue a través de redes sociales.

Los graduados se desempeñan en ocupaciones relacionadas con la carrera estudiada; según la clasificación de la encuesta del OLE en actividades como el arte, la cultura, el esparcimiento, el deporte y la educación $\mathrm{n}^{7}$; lo cual es un indicador de la pertinencia del programa, pues se demuestra que hay un amplio campo de acción para el desarrollo de sus actividades profesionales. La actividad económica en la que se desempeñan la mayoría es el área específica de la educación.

Según las respuestas de los egresados, se puede observar una relación directa entre el tiempo de experiencia y el nivel de ingresos, dado que a medida que va aumentando su experiencia, el promedio de sus ingresos también lo hace. Adicionalmente, trabajan en empresas del ámbito local en su mayoría. Se resalta que los graduados expresan el interés de crear empresa, pero su mayor dificultad es la falta de recursos económicos; cabe resaltar que dentro del plan de estudios del programa tienen un área definida en administración aplicada al deporte, que brinda elementos hacia la constitución de empresa. Los egresados consideran que las posibilidades de conseguir trabajo por ser egresados de dicho programa son buenas, es decir, que están seguros que cuentan con buena aceptación y reconocimiento en el mercado laboral.

En cuanto las competencias laborales generales, las que presentaron mayor satisfacción desde la percepción de los graduados fueron el "comprender la realidad que lo rodea y asumir una cultura de convivencia" y "aplicar valores y ética profesional en el desempeño laboral", esto se puede explicar desde los lineamientos pedagógicos de la Universidad Santo Tomás, que desde su misión y el Proyecto Educativo Institucional buscan una formación integral del estudiante y se evidencia manteniendo como eje transversal del currículo la formación ética ${ }^{12}$.

La contribución que la Universidad hace a su contexto social, cultural, político y económico, fundamentado en el desarrollo científico que desde sus actividades sustantivas son la docencia, la investigación y la proyección social, responden a los desafios de la educación, a lo que se denomina perti- nencia, y que es consignado en el plan de desarrollo de la educación en Colombia como la calidad que debe evidenciar cualquier institución o universidad en sus diferentes niveles garantizando el desarrollo humano y la educación para el trabajo.

Dentro de las limitaciones de este trabajo se percibe la baja participación de los egresados, pues solamente se encuentran representados un $28,6 \%$, por ello estos resultados no se pueden extrapolar a la totalidad de la población de graduados y deben interpretarse con precaución. Por otra parte, en el presente estudio solo se ha tenido en cuenta la percepción de los egresados, pero en términos de pertinencia del programa, valdría la pena explorar en posteriores estudios la percepción de los empleadores y de la misma comunidad con que se relacionan los egresados, para de esta manera retroalimentar los puntos de vista de diferentes sectores de la comunidad.

Este es el primer estudio realizado con los egresados del programa en que se recopila información valiosa con respecto a su situación laboral, así como de la experiencia que han tenido en el contexto y su análisis de la utilidad de las competencias formadas durante su proceso de formación. Conocer que desde la percepción de los graduados de un programa, las competencias laborales cumplieron con sus expectativas, enmarca la propuesta curricular como un programa de alta calidad, pues hablar de la pertinencia de una institución que responde a los retos, a las necesidades y problemas de determinado sector, la hacen visible y evidencian las orientaciones de sus acciones sustantivas congregadas en el currículum y dinamizadas por su plan de estudios.

\section{CONCLUSIÓN}

Se evidencia una clara misión de la Universidad por sus propósitos de imprimir en sus egresados los valores y la ética profesional, considerando la formación como compromiso con la sociedad, declarando también su perfil desde los referentes disciplinares y profesionales.

\section{Agradecimientos}

Los autores agradecen a la Universidad Santo Tomás por el apoyo prestado en el desarrollo del proyecto y a la formación de uno de sus investigadores.

\section{REFERENCIAS}

1. Malagón LA. La pertinencia en la educación superior: elementos para su comprensión. Rev Educ. Sup. 2003;32(3):115-138.

2. Ministerio de Educación. [Internet] Plan Decenal de Educación 2006-2016. Capítulo 1. Fines de la educación y su calidad en el siglo XXI. [Acceso 17 de noviembre de 2012]. 
Recuperado a partir de: http://www.mineducacion.gov. co/1621/article-241792.html

3. Consejo Nacional de Acreditación (CNA). [Internet] Calidad y pertinencia, 2016; [Acceso agosto 8 de 2014. Recuperado a partir de: http://www.cna.gov.co

4. Chiavenato I. Introducción a la teoría general de la administración. 7a. edición. México: McGraw-Hill, 2006.

5. Observatorio Laboral para la Educación (OLE). [Internet]. Estudiantes y graduados. [Acceso 2014 septiebre 16]. Recuperado a partir de: http://www.graduadoscolombia. edu.co/html/1732/w3-propertyvalue-36276.html.

6. Sampieri HR, Fernández C, Lucio BP. Metodología de la Investigación. 4a- ed. México: McGraw-Hill, 2006.

7. Observatorio Laboral para la Educación (OLE). [Internet]. Administrador de encuesta de seguimiento a graduados. [Acceso 2014 septiembre 22]. Recuperado a partir de: http://www.graduadoscolombia.edu.co/html/1732/article-142277.html

8. Fleming J, Martin AJ, Hughes HZ. Maximizing work integrantes. Learning experiences through identifying graduate competencies for employability: a case Study of sport Studies in higher education. Asia-Pacific Jour Coop Educ. 2009;10(3):189-201.
9. Agencia Nacional de Evaluación de la Calidad y Acreditación. ANECA. Libro Blanco. Título de grado en ciencias de la actividad física y del deporte. [Internet]. Madrid-España. [Acceso 2014 octubre 10]. Recuperado a partir de: http://www.aneca.es/var/media/150296/libroblanco_deporte_def.pdf

10. Alberti G, Villena JL, Peluffo MB. Desafíos y oportunidades de la empleabilidad de los egresados universitarios en el contexto internacional. México: McGraw-Hill, 2013.

11. Teichler U. Graduados y empleo: Investigación, metodología y resultados. Madrid-España: Miño y Dávila editores, 2005.

12. López BI, López NM. Lineamientos pedagógicos institucionales. Centro de Estudios en Educación. Colombia: Universidad Santo Tomás, 2010.

\section{Correo electrónico de los autores}

Mike William Barreto: mike.barreto@ustabuca.edu.co, Paula Camila Ramírez: paula.ramirez@ustabuca.edu.co

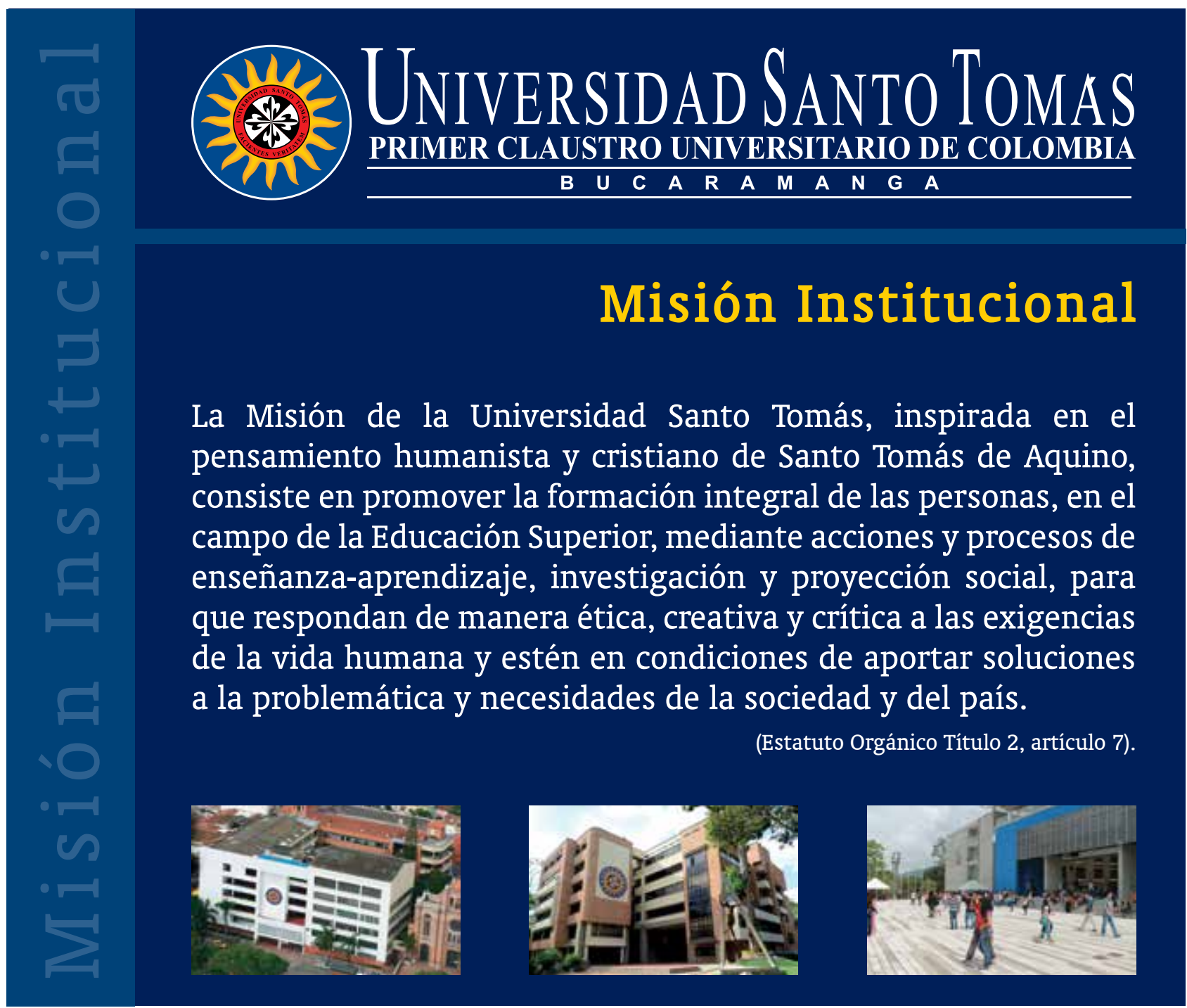

